\title{
Older Adults' Use of Online Neighborhood Social Networks: Perceptions, Challenges and Effects
}

\author{
Pascal Vogel \\ University of Hamburg \\ pascal.vogel@uni-hamburg.de
}

\author{
Christian Grotherr \\ University of Hamburg \\ christian.grotherr@uni-hamburg.de
}

\author{
Franziska von Mandelsloh \\ Hamburg University of Applied Sciences \\ franziska.vonmandelsloh@haw-hamburg.de
}

\author{
Uta Gaidys \\ Hamburg University of Applied Sciences \\ uta.gaidys@haw-hamburg.de
}

\author{
Tilo Böhmann \\ University of Hamburg \\ tilo.boehmann@uni-hamburg.de
}

\begin{abstract}
Online social networks (OSNs) have demonstrated potential for enabling older adults to remain socially connected and for counteracting social isolation and loneliness. With older adults preferring to age in place, their local community and neighborhood gain in importance. Online neighborhood social networks (ONSNs) are a novel type of OSN aimed at connecting local communities by facilitating social interaction, information sharing and peer support among neighbors. With a focus on trust and privacy, local relevance and integration with local organizations and institutions, they might be particularly well suited for the needs of older adults. We investigate the relationship between older adults and ONSNs by analyzing usage data, an online survey and interviews with users of an ONSN active in two urban neighborhoods in Germany. Our findings show that the case ONSN was successful in facilitating communication between neighbors and in promoting participation in community life for older adults.
\end{abstract}

\section{Introduction}

Improved longevity and declining fertility are causing a profound worldwide demographic change, with many countries' populations aging at an unprecedented pace. The United Nations estimate that in the year 2050, one in five people globally will be aged 60 years or older [1]. This development is expected to exert pressure on health care, social security and pension systems [2]. Due to the agglomeration of older adults in cities, urban areas are particularly affected by population aging [3]. Cities are responding by aiming to become age-friendly, increasingly catering to the specific needs of older adults regarding accessibility, security and participation [4]. With rising age, one's immediate surroundings, the neighborhood, gain in importance and become the preferred range of activities [5]. Older adults prefer to age in place and those aged 70 or older spend 80 percent of their time in their home or neighborhood [6]. Being able to interact with and access public and private actors, resources and infrastructure in their neighborhood, determines the experience of inclusion and exclusion for these individuals [7]. At the same time, rising age and the accompanying significant life events such as retirement, death of a spouse or loss of motor function put older adults at risk of suffering from social exclusion, social isolation and loneliness [8].

Digital technologies can play an important part in providing innovative solutions for alleviating the challenges associated with population aging [9]. Among these, online communities and online social networks (OSNs) have presented themselves as viable means for addressing some adverse outcomes of population aging, such as social isolation and loneliness, a lack of social support and a lack of social participation [10-12]. However, many older adults are met with obstacles when using OSNs, including a lack of functional capacity, relevant content or privacy concerns [12].

Online neighborhood social networks (ONSNs) are a novel type of online social network that focuses on improving the well-being of local communities by affording functionality such as information sharing, social interaction, peer support and access to offerings of local organizations and institutions [13]. By limiting access to neighbors inside a delineated geographic area, interactions on ONSNs take place in a community of trust [14]. In recent years, platforms such as Nextdoor (nextdoor.com) or nebenan (nebenan.de) have enjoyed rising popularity $[15,16]$ and are exhibiting promising effects such as increased 
neighborly communication $[13,17]$. By emphasizing locally relevant information, neighbor-provided peer support and with a focus on trust and privacy, they may be particularly well suited for the preferences of older adults. While there are first studies presenting ONSN-like artifacts $[17,18]$, research investigating the relationship between older adults and ONSNs remains scarce, particularly regarding their long-term evaluation $[13,17]$.

To address this research gap, this paper investigates how older adults perceive ONSNs in comparison to other OSNs, how they are affected by ONSN use and what challenges they face regarding adoption and use. We analyze primary data collected from an ONSN called MyNeighbors, which is part of an ongoing research project conducted by the authors $[19,20]$. For this purpose, we leverage platform activity data and conduct an online survey as well as semi-structured interviews with platform users. The findings show that the MyNeighbors ONSN enabled neighborly communication and fostered participation in local community activities for older adults. For the purpose of this research paper, we define older adults as individuals aged 65 years and older [1]. The remainder of this paper is structured as follows. In Section 2, we present related work on ONSNs as well as older adults' use of OSNs. Section 3 outlines our research approach and activities. We present the results of our data analysis in Section 4 and provide an interpretation and discussion of their implications in Section 5. We conclude with a summary, limitations and an outlook on future research.

\section{Related Work}

\subsection{Older adults' usage of online social networks}

Online social networks are commonly defined as "web-based services that allow individuals to (1) construct a public or semi-public profile within a bounded system, (2) articulate a list of other users with whom they share a connection, and (3) view and traverse their list of connections and those made by others within the system" [21, p. 211]. Among older adults, the usage of OSNs is increasing steadily. In 2016, more than $67 \%$ of Americans aged 65 and older had broadband internet access and more than $40 \%$ used at least one social media site, up from $53 \%$ and $34 \%$ respectively in 2012 [22-24].

OSN adoption varies widely among older adults in the same age group, determined by factors such as education, income or employment status and history $[25,26]$. The adoption of OSNs by older adults is hindered by age-related changes such as a decline in coordination skill, memory or declining vision [12, 27]. Further inhibiting factors for OSN use by older adults are negative attitudes towards OSNs due to critical media coverage, a lack of formal conduct on OSNs, a reluctance towards self-disclosure, complex user interfaces, a lack of content perceived as personally relevant and a lack of control of personal data [12].

For older adults, the online environment afforded by OSNs can serve as a source of social connectedness and a viable means of social interaction for individuals lacking face-to-face connections [28, 29]. Compared to young adults, older adults are more successful in deriving social connectedness from OSNs and less likely to experience negative effects of OSN use [12, 29, 30]. Furthermore, OSNs can serve as a complementary source of social support for older adults and can afford them a feeling of control and self-efficacy $[10,12]$. By serving as an everyday context for cognitive stimulation and information processing, OSNs can positively impact the cognitive ability of older adults [31]. Previous research has demonstrated the potential and feasibility of leveraging OSNs in general and neighborhoodfocused online communities in particular to improve the well-being of older adults $[10,13]$. However, as of yet, research has not confirmed this potential via a long-term and naturalistic evaluation.

\subsection{Online Neighborhood Social Networks}

Beginning in the 1980s, community informatics projects such as the Blacksburg Electronic Village leveraged the diffusion of broadband internet technology to establish online communities aimed at the inhabitants of specific, geographically delimited areas $[32,33]$. Segmentative network effects have led to the formation of similar online communities on OSNs such as Facebook in the form of groups [34]. In recent years, dedicated OSN platforms for local communities have experienced steep growth. These online neighborhood social networks can be defined as OSNs whose intended audience comprises the inhabitants of one or more spatially delimited neighborhoods and whose thematic and functional focus lies on issues related to these neighborhoods [14]. Examples of popular ONSNs include U.S.-based Nextdoor with more than 27 million monthly active users and 236,000 active neighborhoods globally, Germany platform nebenan with 1.6 million users across Europe or Neighbourly with 830,000 users in New Zealand [16, 35].

Core capabilities of ONSN platforms entail enabling information sharing, improving social 
connectedness and social participation, establishing a peer support network and integrating with local organizations and institutions [13]. Most ONSN platforms share a common set of functionality [14], including a neighborhood-wide activity stream for sharing local news, making announcements, asking questions or recommendations as well as requesting and providing peer-support. Furthermore, neighbors possess an individual profile and can communicate via chat or direct messaging. Other common features of ONSN platforms include neighborhood calendars, marketplaces, public and private groups, business profiles and other minor features.

ONSNs differ from traditional OSNs by separating users into isolated sub-communities based on their place of residence, usually enforced via identity and address verification mechanisms ranging from inperson ID checks to sharing one's device location. The term neighborhood is multi-faceted and notoriously hard to define, characterizations ranging from an area's socio-economic attributes to following geographic points of reference or municipal boundaries [36, 37]. Similarly, ONSNs take a variety of approaches to delimit their in-platform neighborhoods, including adhering to municipal boundaries, radius-based approaches, crowdsourced user-defined boundaries or algorithmically generated boundaries [14].

Recently, the peer support capabilities of ONSNs have found application during the COVID-19 pandemic [38], allowing local volunteers aiming to provide services such as household and shopping assistance to be matched to individuals in need in their neighborhood. Previous research on ONSNs has identified promising effects such as increased neighborly communication and activity, intergenerational communication and sense of community [13, 17, 18, 39] and a number of studies concerned with the design and evaluation of ONSNlike artifacts can be identified in the literature [10, 13, 17, 20, 40]. Studies explicitly investigating the relationship between older adults and ONSNs remain scarce $[10,13,17]$.

\section{Methodology}

To investigate the relationship between older adults and ONSNs, we leverage empirical data collected in the context of the MyNeighbors ONSN. We utilize three sources of qualitative and quantitative data: platform usage data, an online user survey as well as semi-structured interviews. MyNeighbors is developed and evaluated by the authors as part of an ongoing design science research project in the field of healthy aging and connected communities [13, 20] allows for deep access and insights into platform activity and content.

\subsection{The MyNeighbors ONSN}

The MyNeighbors ONSN is being piloted in two case neighborhoods in a large German metropolitan area and has around 140 verified neighbors at the time of writing. The platform's features are similar to the common feature set described in Section 2.1. Neighbors verify their identity in person or by receiving a verification code via physical mail and are assigned a sub-community based on their address. They contribute to a neighborhood-wide activity stream by creating posts of different categories, communicate via direct messages, can access a neighborhood calendar of local events and are provided with a list of local organizations and their offerings. Each neighbor possesses an individual profile page where he or she can provide a profile image, contact information as well as a selfdescription and interests. MyNeighbors provides a variety of configuration options for specifying which personal data such as name, address and contact information are visible to other users.

A neighborhood directory provides an overview of all verified neighbors in one's neighborhood. Furthermore, MyNeighbors is integrated with a professional neighborhood management service which acts as online and offline community management, a health counseling service as well as smartphone training classes for senior citizens. Neighbors are notified of important events on the platform via configurable email notifications. From a technical perspective, MyNeighbors is a web-based platform developed using the Django web development framework (djangoproject.com) and provides a responsive user interface for desktop and mobile devices.

\subsection{Data collection \& analysis}

We collect MyNeighbors platform usage data in the one-year timespan between June $15^{\text {th }}, 2019$ and June $15^{\text {th }}, 2020$. Data is collected using the Matomo open-source analytics software as well as the capabilities of the MyNeighbors platform itself. We analyze the collected data using the Microsoft Power BI data analysis software. Among the analyzed data are both user activity (e.g., logins, visits, used software features) and contributions (e.g., posts, private messages, number of registered users). We exclude usage data produced by the authors from our analysis. 
The online user survey was active from December 2019 until the end of March 2020. 28 MyNeighbors users participated in the survey $(21 \%$ of 131 verified users at the time of survey closing). The survey contained a total of 50 questions, structured based on the base capabilities of the MyNeighbors platform (i.e., social interaction, information sharing, peer support, and others) but also questions regarding perceptions of privacy, ease of use and usefulness as well as a set of demographic questions. The majority of questions were based on a four-point Likert scale (agree, somewhat agree, somewhat disagree, disagree), complemented by multiple-choice and open-ended questions. The survey was presented to verified MyNeighbors users directly via the ONSN platform upon login and distributed as part of an email newsletter. We perform eight semi-structured interviews with MyNeighbors users from both case neighborhoods in December and January 2020. Interviewees were approached via private message on the MyNeighbors platform or via local events in the case neighborhoods. Interviews took place in the subjects' homes, were documented using researcher field notes and refined using audio recordings. The semi-structured interviews followed a predefined interview guide containing open questions regarding general neighborhood life, MyNeighbors platform usage as well as demographic characteristics of participants. Direct quotes were carefully translated from German into English. By combining several data collection and analysis approaches (platform usage data, online survey and qualitative interviews), we balance the limitations of one evaluation element with the strengths of the others. Consequently, we can triangulate findings and can confirm usage patterns across multiple data sources.

\section{Findings}

\subsection{Platform usage}

4.1.1. Overview. At the end of our data collection period (June $\left.15^{\text {th }}, 2020\right), 136$ users were registered and verified for the MyNeighbors ONSNs platform (146 including unverified users) in the two case neighborhoods. Three groups of users can be distinguished on MyNeighbors. Neighborhood managers, representing the smallest group with six members, act as online community managers and facilitators for the other user groups. They possess certain administrative rights for curating platform content and user verification. Professionals represent the second largest group, with 38 users.
Table 1. MyNeighbors users age distribution

\begin{tabular}{ccccc}
\hline Age & $\mathbf{N}$ & $\mathbf{P}$ & $\mathbf{N m}$ & $\sum$ \\
\hline $18-34$ & $11(12 \%)$ & $17(45 \%)$ & $2(33 \%)$ & $30(22 \%)$ \\
$35-44$ & $11(12 \%)$ & $12(32 \%)$ & $1(17 \%)$ & $24(18 \%)$ \\
$45-54$ & $12(13 \%)$ & $2(5 \%)$ & $1(17 \%)$ & $15(11 \%)$ \\
$55-64$ & $22(24 \%)$ & $7(18 \%)$ & $2(33 \%)$ & $31(23 \%)$ \\
$65+$ & $36(39 \%)$ & - & - & $36(26 \%)$ \\
\hline$\sum$ & 92 & 38 & 6 & 136 \\
$\varnothing$ age & 58,6 & 37,5 & 45,8 & 52,2 \\
\hline $\mathrm{N}=$ neighbors; $\mathrm{P}=$ professionals; $\mathrm{Nm}=$ neighborhood managers
\end{tabular}

These include members of organizations and institutions which are active in the case neighborhoods, such as clubs, churches, nonprofits and health service providers. Finally, neighbors are private individuals using the MyNeighbors platform. As they are at the center of our research, the analysis in the following Sections 4.1.2. to 4.1.4. is limited to data of the neighbor user group (i.e., excluding professionals and neighborhood managers) unless otherwise stated. Table 1 provides an overview of the age distribution across these user groups.

4.1.2. User activity. We measure user activity on the MyNeighbors platform based on the number of logins and requests made by its users. Logins constitute visits to the website where the username and password are actively and successfully submitted. It must, however, be noted that not each visit to the MyNeighbors platform necessitates a new login as session information is stored across multiple visits. We evaluate HTTP GET requests to gain a more finegrained measure of actions performed on the website subsequent to login. Figure 1 provides an overview of logins and requests made per user by age group.
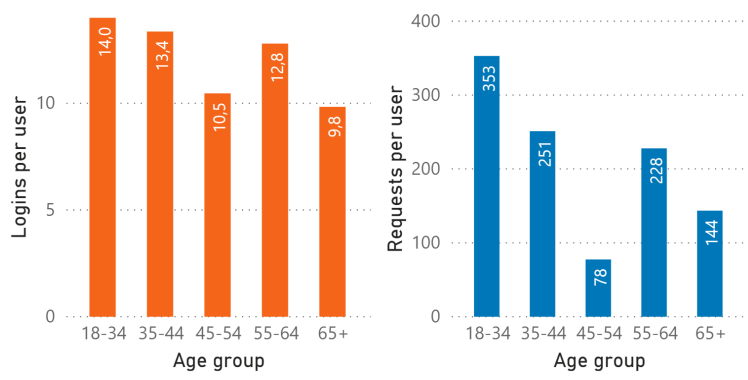

Figure 1. Logins and requests per user by age group

Across all age groups, the average number of logins per user was 12.1. Older adults logged in an average of 9.8 times, representing the group with the lowest number of logins, coming close only to the group of users 45 to 54 years of age with 10.5 logins. 
Regarding actions on the MyNeighbors platform, older adults ranked below the average of 210 requests across age groups, performing 144 requests per user on average. In contrast, the youngest group of users, aged 18 to 34 years, performed the highest number of actions on the platform with 353 requests per user.

4.1.3. User contributions. A total of 658 posts were published on the MyNeighbors platform during our data collection period. Of these posts, 282 were created by neighborhood managers, 188 by professionals and 188 by neighbors. The 188 posts created by neighbors included 141 events, 38 announcements, five offers, three requests and one question. Users of all user groups communicated with each other directly via 397 private messages (236 sent by neighbors), wrote 140 comments ( 83 published by neighbors) and received 6296 email notifications. Figure 2 provides an overview of the content generated per user on MyNeighbors by age group.

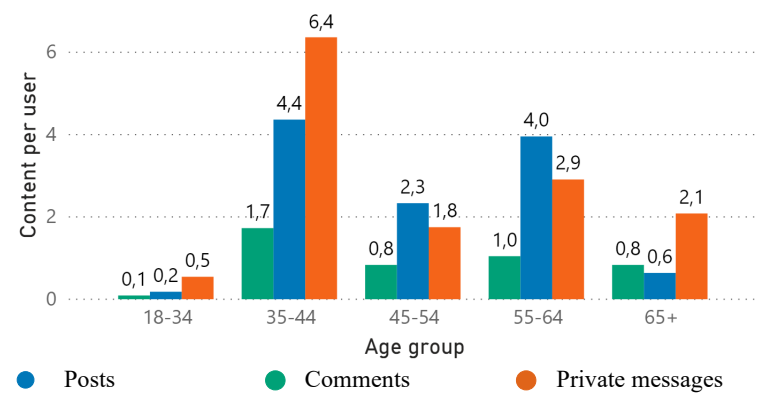

Figure 2. Generated content per user by age group

With an average of 0.6 posts per user, older adults created the second-lowest number of posts on the MyNeighbors activity stream out of all age groups. They created 2.1 private messages per user, being surpassed by the 35 to 44 and 55 to 65 year old users. Regarding comments made to posts, older adults rank identical to the 45 to 65 old users with 0.8 posts on average and are ahead of the 18 to 34 years user group.

4.1.4. Functionality usage. To illustrate functionality usage, we group the requests measured as described in 4.1.2. by the platform functionality they relate to. Requests not directly attributable to a platform functionality were excluded. With 39 requests per user, the neighbor directory as well as individual neighbor profiles were the most used functionality of MyNeighbors across age groups, followed by posts with 34 requests and the MyNeighbors calendar with 28 requests. Groups and the MyNeighbors offerings directory seem not to have attracted much attention from users, with only 3.4 and 3 requests per user respectively. Figure 3 provides a more detailed look at feature usage per user by age group based on requests made in the context of a specific functionality.

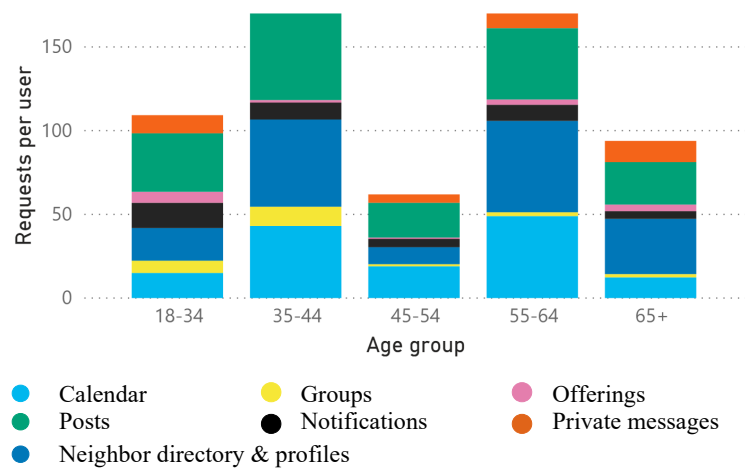
Figure 3. Functionality usage per user by age
group

Generally, the majority of requests performed by older adults on MyNeighbors is associated with an above-average activity related to private messages as well as activity related to posts and the neighbor directory or profiles. While the previous Section 4.1.3. showed that older adults did generate few posts compared to other age groups, a considerable share of their on-platform actions were related to reading posts and visiting the post overview on the MyNeighbors activity stream. Despite not sending the most messages on MyNeighbors compared to other age groups as presented in Section 4.1.3., older adults performed more actions related to private messages than any other age group. Older adults, furthermore, did not perform many actions related to the MyNeighbors calendar compared to other age groups.

\subsection{Online user survey}

4.2.1. Overview. The 28 survey respondents had an average age of 60.2 years, ranging from 44 to 82 years. Participants were $43 \%$ female and $54 \%$ male, with an average household size of 1.7. Respondents estimated that they had used MyNeighbors for an average of 4.1 months prior to completing the survey. We included one partially completed survey in the following analysis.

4.2.2. Social connectedness, social participation and information sharing. The majority of respondents indicated that they valued communication and exchange with neighbors via the MyNeighbors platform ( $25 \%$ agree, $36 \%$ somewhat agree). In part, respondents were also successful in making new acquaintances via MyNeighbors (21\% agree, 21\% 
somewhat agree). However, respondents did not generally meet more frequently with neighbors since using MyNeighbors (7\% agree, 14\% somewhat agree) and only some were able to meet neighbors with matching interests ( $11 \%$ agree, $25 \%$ somewhat agree). However, based on responses, the platform was successful in driving neighbors to volunteer in their neighborhood (36\% agree, 29\% somewhat agree).

Respondents possessed a positive perception of information-sharing capabilities of MyNeighbors. Most reported that MyNeighbors had helped them to be better informed regarding events and offerings in the neighborhood (71\% agree, 18\% somewhat agree) and neighborhood life in general $(50 \%$ agree, $39 \%$ somewhat agree).

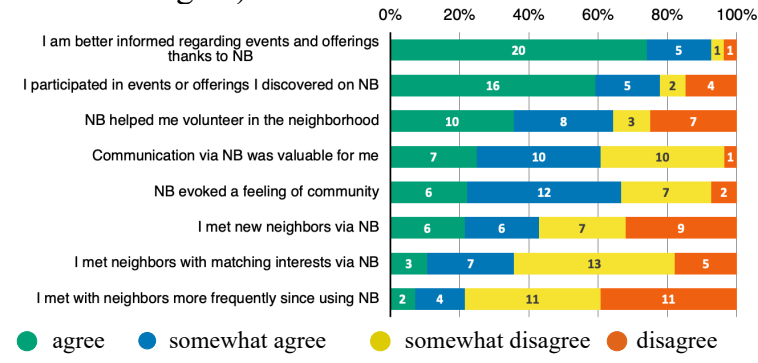

Figure 4. Social connectedness, social participation and information sharing

Respondents acted on the received information and participated in events and offerings discovered via MyNeighbors (57\% agree, 18\% somewhat agree). For some respondents, MyNeighbors was able to evoke a feeling of community (21\% agree, $43 \%$ somewhat agree). Figure 4 provides a detailed overview of responses related to social connectedness, social participation and information sharing.

4.2.3. Peer support. Peer support presented itself as a multi-faceted issue based on survey responses. Respondents reported a strong willingness to provide assistance to others (35\% agree, 53\% somewhat agree) and would also request assistance themselves, if in need (32\% agree, 39\% somewhat agree). However, based on responses, they did not request assistance via MyNeighbors as of yet (71\% disagree, 11\% somewhat disagree) and most respondents did not get the opportunity to assist others (25\% agree, $11 \%$ somewhat agree, $61 \%$ did not have the opportunity).

4.2.4. Motivators and obstacles for platform use. Respondents indicated that their main motivation for using MyNeighbors lay in staying up-to-date with current neighborhood life, including local events and offerings. Furthermore, maintaining existing relationships with other neighbors represented a motivating factor while building new relationships played a minor role. According to respondents, the most motivating platform features were posts by neighborhood managers (considered motivating by $71 \%$ ), followed by posts by other neighbors $(57 \%)$, the neighborhood calendar (54\%) and private messages between neighbors $(18 \%)$.

A lack of new platform content and perceived activity on the platform, i.e., its liveliness, represent obstacles to MyNeighbors usage. However, responses suggest that technical issues or a lack of relevance of the available content are not significant obstacles. Obstacles mentioned via free-text response under "Others" include the lack of native mobile apps and a general lack of free time to commit towards using MyNeighbors. Table 2 presents an overview of motivators and obstacles for MyNeighbors use.

\section{Table 2. Platform use: motivators \& obstacles}

\begin{tabular}{|c|c|c|c|}
\hline \multirow{9}{*}{ : } & Information on local offerings & 23 & $82 \%$ \\
\hline & Information on local events & 21 & $75 \%$ \\
\hline & Staying up-to-date & 19 & $68 \%$ \\
\hline & Maintaining local social ties & 11 & $39 \%$ \\
\hline & Finding specific information & 8 & $29 \%$ \\
\hline & Forming new local social ties & 4 & $14 \%$ \\
\hline & Finding local organizations & 4 & $14 \%$ \\
\hline & Boredom/need for entertainment & 2 & $7 \%$ \\
\hline & Others & 2 & $7 \%$ \\
\hline \multirow{7}{*}{$\begin{array}{l}\frac{\pi}{0} \\
\frac{\pi}{0} \\
\frac{\tilde{\sigma}}{0}\end{array}$} & Lack of participants & 9 & $32 \%$ \\
\hline & Others & 9 & $32 \%$ \\
\hline & Lack of activity & 7 & $25 \%$ \\
\hline & Forget to check for updates & 7 & $25 \%$ \\
\hline & Content not current & 4 & $14 \%$ \\
\hline & Content not relevant & 1 & $4 \%$ \\
\hline & Technical issues & 1 & $4 \%$ \\
\hline
\end{tabular}

4.2.5. Ease of use and usefulness. In comparison to other OSNs, respondents agreed $(50 \%)$ or somewhat agreed (25\%) that MyNeighbors was more suitable for their needs. Similarly, respondents agreed $(21 \%)$ or somewhat agreed $(57 \%)$ that NeigborBook was more clearly designed than other OSNs.

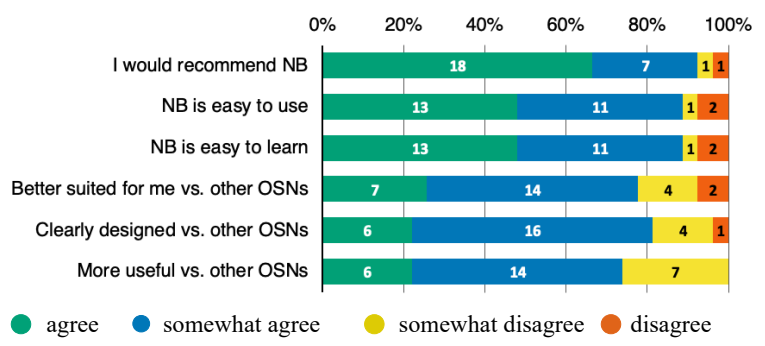

Figure 5. Ease of use and usefulness

Generally, the majority of users considered MyNeighbors as useful (21\% agree, 50\% somewhat agree) and described it as being easy to use and learn (46\% agree, 39\% somewhat agree). Overall, most 
users would recommend MyNeighbors (64\% agree, $25 \%$ somewhat agree). Figure 5 provides a detailed overview of responses related to the platform's ease of use and usefulness.

4.2.6. Trust and privacy. Data privacy represented an important issue for respondents. Overall, they considered their data to be in good hands with MyNeighbors (32\% agree, 61\% somewhat agree). Furthermore, respondents indicated that they trusted MyNeighbors more with regards to data privacy than commercial social networks (43\% agree, $43 \%$ somewhat agree). Regarding preferences for sharing one's name, address and profile image, opinions were split. $43 \%$ of participants preferred seeing the full names of other users on MyNeighbors (e.g., John Doe), while $54 \%$ preferred a partly anonymized version (e.g., John D.). Respondents were largely satisfied with knowing that other users live in the same neighborhood $(50 \%)$, some preferring a more precise location based on the street name $(29 \%)$ or street name and number (18\%). Regarding profile images, a slight majority of respondents agreed or somewhat agreed that it was important to be able to see profile images of other users (21\% agree, $36 \%$ somewhat agree).

\subsection{Semi-structured interviews}

4.3.1 Overview. The eight interviewees had an average age of 68 , ranging from 47 to 84 . They had lived in their neighborhood for an average of 14 years and were evenly split between male and female. Six out of eight interviewees were retired while two were employed part-time and lived in households of 1.25 on average. Six out of eight interviewees accessed MyNeighbors via both smartphone as well as desktop or laptop computer, the other two interviewees relying on only one of these devices. They had been members of MyNeighbors between 2 weeks and eight months prior to the interview taking place.

4.3.2. Social connectedness, social participation and information sharing. According to interviewees, the MyNeighbors platform was able to help them in staying up-to-date with current neighborhood life and a number of interviewees were able to identify and participate in local events via the MyNeighbors platform. Mentioned examples include cooking classes, gymnastics for seniors, meeting for coffee, smartphone training, health counseling or board game afternoons. One interviewee reported publishing her own events to the MyNeighbors calendar in order to attract participants. The MyNeighbors platform was characterized by one interviewee as follows:
For me it is a platform for the [case] neighborhood on which people who live here can meet, make contact and exchange help, information, anything really.

Interviewees described the MyNeighbors platform as a complement but not a substitute for in-person interaction. However, many interviewees saw MyNeighbors as a valid means of initiating new social connections, which could then be further developed offline. Connections would not only arise via direct communication with other MyNeighbors users but also by meeting new neighbors in the context of events discovered via the platform's calendar.

4.3.3. Peer support. The topic of peer support was discussed animatedly by interviewees. Most considered establishing a platform-supported local peer support network as a feasible and commendable undertaking. Similarly, most interviewees would be ready to render assistance to neighbors if necessary, in some cases depending on how well the required assistance fits into their skillset or schedule. One interviewee considered peer support among the inhabitants of individual buildings as a promising scenario as trust among these proximate neighbors would likely be higher than between unknown neighbors in the neighborhood. Regarding using MyNeighbors to receive assistance with household tasks from neighbors one interviewee stated:

That is definitely a possibility, how well it works will depend on the actual human relationship. Such a platform is a starting-point I could hook into. [...] Right now help comes via friends, not via neighbors. [...] I wonder how reliable I could get what I need over certain periods of time [via MyNeighbors].

However, interviewees unanimously reported not yet having requested assistance via the MyNeighbors platform and not having become aware of any requests for assistance they could have answered via the platform. Some interviewees also expressed doubts that those who could really benefit from local peer support would likely not possess an internet-connected device and, therefore, could not be reached effectively.

4.3.3. Motivators and obstacles for use. Interviewees expressed a variety of motivations for using the MyNeighbors platform. Curiosity towards the platform in general, as well as the potential of interacting with neighbors from the immediate neighborhood were mentioned frequently. Furthermore, being able to remain in the loop regarding local events via the MyNeighbors calendar represented an important motivator for interviewees, particularly when asked regarding motivators for 
continued and regular platform usage. Other motivators included wanting to counteract a feeling of social exclusion, being able to look up offerings of local organizations and gaining a novel use-case for smartphone usage. Almost all interviewees described the MyNeighbors platform as easy to use and quick to learn. Some reported that personal instruction during smartphone classes or by neighborhood managers helped them to get started using the platform. The main obstacle for use was described as a lack of diffusion of MyNeighbors in the case neighborhoods and a resulting lack of perceived platform activity or liveliness. Interviewees considered further marketing activity as necessary to increase platform usage.

4.3.4. Trust and privacy. Interviewees had a positive perception of trust and data privacy in relation to MyNeighbors. Several interviewees mentioned that the identity and address verification mandated by MyNeighbors increased their trust in the platform as these mechanisms ensured only real neighbors were present. Generally, data privacy was an important issue for interviewees and being able to trust the MyNeighbors platform was an important condition for using it. Interviewees expressed that trust in the MyNeighbors ONSN was strengthened by a university, perceived as a reputable public institution, acting as the platform provider due to malicious commercial interests being deemed unlikely. One interviewee expressed:

[MyNeighbors] seems to have a reputable background. The university takes care of it and it's checked personally if [users] are really in the neighborhood. I know there's a bunch of safety precautions to make sure that the pot's content fits the label.

Regarding the use of profile pictures, interviewees largely considered them as valuable as they assisted in recognizing known neighbors, facilitated getting to know new neighbors and generally sparked interest in visiting user profiles.

\section{Discussion}

MyNeighbors was generally successful in attracting older adults, with individuals aged 65 and older constituting the largest age group among users. Assessing the platform usage of older adults requires differentiated consideration. The analyzed data shows that older adults did not contribute large amounts of publicly visible user-generated content such as posts to the platform, creating the smallest number of posts per user out of all age groups. They did, however, interact with the posts of other users via comments at a rate similar to other age groups. Regarding direct communication via private messages, older adults performed more platform actions on average than any other age group and created more messages than some younger age groups. Similarly, they performed more on-site actions based on requests than users aged 45 to 54 years and came close to the 18 to 34 demographic. For an age group typically hard to engage via online platforms [25], the data shows a surprising activity.

Consequently, while younger user groups acted as producers of public content, older adults leveraged MyNeighbors mainly for the consumption of this content and for private communication. The observed lack of public self-disclosure is in line with previous research showing that older adults are hesitant to openly communicate on OSNs and have less need for self-portrayal compared to younger user groups [12]. It can be speculated that the high activity related to private messages sent by older adults may in part be the result of a lower diffusion of messenger apps for direct communication such as WhatsApp among older adults compared to younger neighbors.

Access to locally relevant information, particularly regarding local events and general neighborhood life, presented itself as the main driver of platform usage cross usage data, online survey and interviews. Based on their combined on-site actions, posts and the MyNeighbors calendar represent the most used functionality. Survey responses and interviews indicated clearly that MyNeighbors helped them to stay up-to-date with current neighborhood life and that they actively participated in events discovered via MyNeighbors. Partaking in these events and offerings can also be considered as positive regarding the social connectedness and participation of neighbors. Almost half of survey respondents reported meeting new neighbors via MyNeighbors, a considerable number especially considering the high age of respondents and their long residence in the case neighborhoods.

Based on our analyzed data, the MyNeighbors peer support network remained largely unutilized. Usage data shows that almost no posts of the category request were made. Similarly, survey respondents and interviewees indicated that they did not request any assistance via MyNeighbors. Conversely, a number of unsolicited offers for assistance could be observed in the usage data, in some cases related to the COVID-19 pandemic. It is unclear if these offers were accepted as communication to this end may have been conducted outside the MyNeighbors platform. Furthermore, survey respondents and interviewees exhibited a high readiness to support neighbors if the need for assistance ever arose but did not have the opportunity to act due to a lack of requests for help. Dissolving this causality dilemma may require external, non-neighbor 
stimulation, for instance via neighborhood managers publishing requests for assistance by proxy and thereby jump-starting the peer support network. Stimulation may also come via specific, novel functionality that provides structure to requests and nudges users to provide peer support.

Based on survey and interview analysis, platform users showed greater trust towards MyNeighbors concerning the handling of their personal data compared to other OSNs. The platform profited from its provider being a public institution, the address and identity verification mechanisms in place, privacy controls as well as real-name usage. Users described MyNeighbors as easy to use compared to other OSNs which may be explained by lower complexity due to a small feature set compared to other OSNs. Smartphone classes offered for older adults in the case neighborhoods also lowered the entry barrier to MyNeighbors and internet-connected devices in general for some users. Although, based on the limited data available, it cannot be determined with certainty if MyNeighbors is better-suited for older adults than other OSNs, the platform does show potential in addressing some obstacles faced by older adults when using OSNs, including complex user interfaces, lack of personally relevant content and concerns with data privacy [12]. The community of trust evoked by neighborhood sub-communities and the resulting smaller audience compared to other OSNs, may also have positively influenced adoption by older adults.

\section{Conclusion and Outlook}

In this study, we investigated the use of ONSNs by older adults based on empirical data collected from the NeigborBook ONSN platform. We analyze platform usage data, an online survey and interviews of platform users. Results show that the ONSN was generally successful in attracting and being utilized by a user base of older adults. We observe that the platform established itself as a useful information sharing medium in the case neighborhoods and served as a means of communication for older adults, who readily interacted with MyNeighbors. It was less successful in establishing a local peer support network among neighbors. Compared to other OSNs, users valued the ease of use as well as focus on trust and privacy of MyNeighbors but were in some cases deterred by a lack of perceived activity and liveliness.

This research furthers the understanding of digital technology use by older adults by providing a detailed perspective on their usage of OSNs and, in particular, ONSNs. We demonstrate the potential of ONSNs to positively impact the social connectedness and participation of older adults and provide insights for the future development of online communities aimed at improving the well-being of older adults in their neighborhood. Furthermore, we present ONSNs as one potential building block of initiatives aiming to create an urban environment that is age-friendly and enables older adults to age actively and in place.

This research is faced with some limitations. Data sample size is limited, collected from a relatively constricted geographic area and prone to selection bias. Therefore, the generalizability of the presented findings to a wider population and neighborhoods with different characteristics has to be considered as limited. The unique context of our case ONSN as part of a larger research project with neighborhood managers, health consultants and smartphone classes also hinders transferability to other settings. In the future, the MyNeighbors ONSN is expected to expand to further case neighborhoods in the same metropolitan area, enabling a comparison between neighborhoods with varying socio-demographic properties. In the long-term, an expansion into other cities, regions or countries could yield further impactful insights on ONSN use by older adults.

\section{Acknowledgments}

This research was funded by the European Regional Development Fund and the City of Hamburg as part of the research project AGQua (https://www.agqua.de).

\section{References}

[1] UN. World Population Ageing Report 2017. United Nations Department of Economic and Social Affairs Population Division, 2017.

[2] Shankar, A., McMunn, A., Banks, J., and Steptoe, A. Loneliness, social isolation, and behavioral and biological health indicators in older adults. Health Psychology, 30, 4 (2011), 377-385.

[3] Leeson, G.W. The Growth, Ageing and Urbanisation of our World. Journal of Population Ageing, 11, 2 (2018), 107115 .

[4] Buffel, T., Phillipson, C., and Scharf, T. Ageing in urban environments: Developing 'age-friendly' cities. Critical Social Policy, 32, 4 (2012), 597-617.

[5] Yen, I.H., Shim, J.K., Martinez, A.D., and Barker, J.C. Older people and social connectedness: how place and activities keep people engaged. Journal of Aging Research, 2012 (2012), 1-10.

[6] Wahl, H.-W., Iwarsson, S., and Oswald, F. Aging Well and the Environment: Toward an Integrative Model and Research Agenda for the Future. The Gerontologist, 52, 3 (2012), 306-316.

[7] Buffel, T., Phillipson, C., and Scharf, T. Experiences of neighbourhood exclusion and inclusion among older people 
living in deprived inner-city areas in Belgium and England. Ageing and Society, 33, 1 (2013), 89-109.

[8] Coyle, C.E., and Dugan, E. Social Isolation, Loneliness and Health Among Older Adults. Journal of Aging and Health, 24, 8 (2012), 1346-1363.

[9] Mostaghel, R. Innovation and technology for the elderly: Systematic literature review. Journal of Business Research, 69, 11 (2016), 4896-4900.

[10] Goswami, S., Köbler, F., Leimeister, J.M., and Krcmar, H. Using Online Social Networking to Enhance Social Connectedness and Social Support for the Elderly. International Conference on Wirtschaftsinformatik, St. Louis, USA, 2010.

[11] Srivastava, S.K., and Panigrahi, P.K. Social Participation among the Elderly: Moderated Mediation Model of Information and Communication Technology (ICT). Communication of the Association for Information Systems, 44 (2019), 698-717.

[12] Leist, A.K. Social Media Use of Older Adults: A MiniReview. Gerontology, 59, 4 (2013), 378-384.

[13] Vogel, P., Jurcevic, N., and Meyer-Blankart, C. Healthy, Active and Connected: Towards Designing an AgeFriendly Digital Neighborhood Platform. European Conference on Information Systems, Stockholm-Uppsala, Sweden, 2019.

[14] Vogel, P., Grotherr, C., Kurtz, C., and Böhmann, T. Conceptualizing Design Parameters of Online Neighborhood Social Networks. International Conference on Wirtschaftsinformatik, Potsdam, Germany, 2020.

[15] Nextdoor. 2019. Nextdoor raises $\$ 123 M$ to accelerate the global power of local. 2019-07-15, https://tinyurl.com/y2enpc71

[16] nebenan. 2020. Zahlen und Fakten zu nebenan.de. 2020-05-29, https://tinyurl.com/yxt7qgsu

[17] Renyi, M., Gündogdu, R., Kunze, C., Gaugisch, P., and Teuteberg, F. The Networked Neighborhood. IEEE International Conference on Engineering, Technology and Innovation, Konstanz, Germany, 2018.

[18] Masden, C.A., Grevet, C., Grinter, R.E., Gilbert, E., and Edwards, W.K. Tensions in Scaling-Up Community Social Media: A Multi-Neighborhood Study of Nextdoor. International Conference on Human Factors in Computing Systems, Toronto, Canada, 2014.

[19] Vogel, P., Grotherr, C., and Böhmann, T. Designing Tool Support for Crowd-Sourced Community Initiatives on Online Neighborhood Social Networks. European Conference on Information Systems, Marrakech, Morocco, 2020.

[20] Grotherr, C., Vogel, P., and Semmann, M. Multilevel Design for Smart Communities: The Case of Building a Local Online Neighborhood Social Community. Hawaii International Conference on System Sciences, Grand Wailea, HI, USA, 2020.

[21] boyd, d.m., and Ellison, N.B. Social Network Sites: Definition, History, and Scholarship. Journal of ComputerMediated Communication, 13, 1 (2007), 210-230.

[22] Pew Research Center. 2019. Social Media Factsheet. 2020-06-18, https://tinyurl.com/s2tc3by

[23] Anderson, M., and Perrin, A. 2017. Technology use among seniors. 2018-10-01, https://tinyurl.com/n2euh9x
[24] Pew Internet Research. 2012. Older adults and internet use. 2020-07-01, https://tinyurl.com/y4122eef

[25] Niehaves, B., and Plattfaut, R. Internet adoption by the elderly: employing IS technology acceptance theories for understanding the age-related digital divide. European Journal of Information Systems, 23, 6 (2014), 708-726.

[26] Hunsaker, A., and Hargittai, E. A review of Internet use among older adults. New Media \& Society, 20, 10 (2018), 3937-3954.

[27] Boll, F., Brune, P., and Gewald, H. Towards Your Parents' Social Network Platform - A User Interface For the Age of Retirement. Hawaii International Conference on System Sciences, Waikaloa Village, HI, USA, 2017.

[28] Sinclair, T.J., and Grieve, R. Facebook as a source of social connectedness in older adults. Computers in Human Behavior, 66 (2017), 363-369.

[29] Grieve, R., and Kemp, N. Individual differences predicting social connectedness derived from Facebook: Some unexpected findings. Computers in Human Behavior, 51 (2015), 239-243.

[30] Barbosa Neves, B., Franz, R., Judges, R., Beermann, C., and Baecker, R. Can Digital Technology Enhance Social Connectedness Among Older Adults? A Feasibility Study. Journal of Applied Gerontology, 38, 1 (2019), 49-72.

[31] Quinn, K. Cognitive Effects of Social Media Use: A Case of Older Adults. Social Media + Society, 4, 3 (2018), $1-22$.

[32] Carroll, J.M., and Rosson, M.B. Developing the Blacksburg electronic village. Communications of the ACM, 39, 12 (1996), 69-74.

[33] Lee, F.S.L., Vogel, D., and Limayem, M. Virtual Community Informatics: A Review and Research Agenda. Journal of Information Technology Theory and Application, 5, 1 (2003), 47-61.

[34] Ilena, M., Ard, H., and Wim, B. On the Development of Online Cities and Neighborhoods: an Exploration of Cumulative and Segmentive Network Effects in Social Media. European Conference on Information Systems, Barcelona, Spain, 2011.

[35] Neighbourly. 2020. Neighbourly shines in a time of coronavirus. 2020-05-23, https://tinyurl.com/y6zq3nry

[36] Sampson, R.J., Raudenbush, S.W., and Earls, F. Neighborhoods and Violent Crime: A Multilevel Study of Collective Efficacy. Science, 277, 5328 (1997).

[37] Payne, W. Welcome to the Polygon: Contested Digital Neighborhoods and Spatialized Segregation on Nextdoor. Computational Culture, 6 (2017), 1-39.

[38] Kummitha, R.K.R. Smart technologies for fighting pandemics: The techno- and human-driven approaches in controlling the virus transmission. Government Information Quarterly (2020), 101481.

[39] Daly, E.M., Dahlem, D., and Quercia, D. The new blocs on the block: using community forums to foster new neighbourhoods. ACM Conference on Web Science, Bloomington, Indiana, USA, 2014.

[40] Antonini, A., Boella, G., Calafiore, A., Salaroglio, C., Sanasi, L., and Schifanella, C. First Life, the Neighborhood Social Network: a Collaborative Environment for Citizens. ACM Conference on Computer Supported Cooperative Work, San Francisco, USA, 2016. 